\title{
CARACTERIZAÇÃO EPIDEMIOLÓGICA DOS CASOS DE SÍFILIS CONGÊNITA NA REGIÃO NORTE DO BRASIL, NO PERÍODO 2014 A 2019
}

\section{ARTIGO ORIGINAL}

ALMEIDA, Carolina Gomes ${ }^{1}$

ÁVILA, Gabriel Pereira ${ }^{2}$

TEIXEIRA, Isabelly Montenegro ${ }^{3}$

RODRIGUES, Raíza Júlia Viana ${ }^{4}$

DIAS, Claudio Alberto Gellis de Mattos ${ }^{5}$

OLIVEIRA, Euzébio de ${ }^{6}$

DENDASCK, Carla Viana ${ }^{7}$

\footnotetext{
${ }^{1}$ Acadêmico do curso de Medicina da Universidade Federal do Pará (UNIFAP).

${ }^{2}$ Acadêmico do curso de Medicina da Universidade Federal do Pará (UNIFAP).

${ }^{3}$ Acadêmico do curso de Medicina da Universidade Federal do Pará (UNIFAP).

${ }^{4}$ Acadêmico do curso de Medicina da Universidade Federal do Pará (UNIFAP).

${ }^{5}$ Doutor em Teoria e Pesquisa do Comportamento (UFPA). Professor e Pesquisador no Instituto Federal do Amapá (IFAP), campus Macapá, AP. Professor e Pesquisador no Programa de Pós-Graduação em Educação Profissional e Tecnológica (ProfEPT), IFAP, campus Santana.

${ }^{6}$ Doutor em Doenças Tropicais (UFPA). Professor e Pesquisador na Universidade Federal do Pará (UFPA), Campus Castanhal, PA.

7 Doutora em Psicanálise (SEMINÁRIO INTERNACIONAL DE TEOLOGIA). Pesquisadora do Centro de Pesquisas e Estudos Avançados (CEPA). Doutoranda em Comunicação e Semiótica (PUC SP).
} 
ARAÚJO, Maria Helena Mendonça de ${ }^{8}$

FECURY, Amanda Alves ${ }^{9}$

ALMEIDA, Carolina Gomes. Et al. Caracterização epidemiológica dos casos de Sífilis Congênita na região norte do Brasil, no período 2014 a 2019. Revista Científica Multidisciplinar Núcleo do Conhecimento. Ano 05, Ed. 12, Vol. 14, pp. 2031. Dezembro de 2020. ISSN: 2448-0959, Link de acesso: https://www.nucleodoconhecimento.com.br/saude/regiao-norte

\section{RESUMO}

A sífilis é uma doença causada pela bactéria Treponema pallidum, adquirida, em grande maioria, por transmissão sexual. A sífilis congênita é uma doença contagiosa e de transmissão vertical (da mãe para o feto). Este estudo teve por objetivo apresentar o número de casos notificados de sífilis congênita na região norte do Brasil, entre os anos de 2014 a 2019, e caracterizar o perfil epidemiológico dos casos. Foi realizada pesquisa descritiva, transversal e retrospectiva, utilizando banco de dados do DATASUS. Houve aumento do número de casos no período avaliado, destacandose o Estado do Amazonas, que apresentou o maior número de notificações. Com relação a realização do pré-natal, uma média de quatro vezes mais mulheres realizam que as que não realizaram. As mulheres com grau escolar materno da $5^{\circ}$ a $8^{\circ}$ série incompleta do ensino fundamental apresentaram maior número de casos da doença. Apesar de a região norte ter uma elevada realização de pré-natal, a maior parte dos casos de sífilis congênita foi diagnosticada somente após o parto, indicando interpretações errôneas quanto aos exames e consequente erro no diagnóstico e

${ }^{8}$ Mestra em Ensino e ciências da Saúde. Professora e pesquisadora na Universidade Federal do Amapá (UNIFAP), campus Macapá, AP.

${ }^{9}$ Doutora em Doenças Tropicais (UFPA). Professora e pesquisadora na Universidade Federal do Amapá (UNIFAP), campus Macapá. Professora e pesquisadora no Programa de Pós- Graduação em Ciências da Saúde (UFPA). Pró-Reitora de Pesquisa e Pós-Graduação na Universidade Federal do Amapá (UNIFAP). 
tratamento. Sendo a forma precoce (surgimento até o $2^{\circ}$ ano de vida) a maior parte do número de casos, sucede-se uma evolução favorável da doença. A escolaridade e a renda parecem ser fatores que influenciam no diagnóstico tardio da doença. Há necessidade do aumento de parceiros tratados, diminuindo a transmissão da sífilis e, consequentemente, da sífilis congênita.

Palavras-Chave: Sífilis congênita, Região Norte, Pré-natal.

\section{INTRODUÇÃO}

A sífilis é uma infecção bacteriana sistêmica, crônica, curável e exclusiva do ser humano. Quando não tratada, evolui para estágios de gravidade variada, podendo acometer órgãos e sistemas do corpo (BRASIL, 2018). O agente causador da sífilis é a bactéria Treponema pallidum, uma espiroqueta, adquirida na maioria dos casos durante relações sexuais. A doença é classificada, de acordo com suas diferentes vias de transmissão, em sífilis adquirida e sífilis congênita (CAVALCANTE et al., 2012, ROCHA et al., 2020).

A sífilis congênita é o resultado da disseminação hematogênica do T. pallidum, da gestante infectada não-tratada ou inadequadamente tratada para o seu concepto, por via transplacentária (CRT, 2016). A transmissão pode ocorrer durante toda a gestação, sendo o risco maior nas gestantes com sífilis primária ou secundária (GUINSBURG; SANTOS, 2010, BARROS et al., 2020).

É uma doença responsável por aproximadamente $40 \%$ das taxas de mortalidade perinatal, $25 \%$ de natimortalidade, $14 \%$ de mortes neonatais (CARDOSO et al., 2018). Além disso, é capaz de acarretar graves consequências para o concepto como complicações sistêmicas, seja na sífilis congênita precoce (acometimento ósseo, neurológico, hematológico e hepatoesplênico), ou tardia (estigmas decorrentes de remodelação e deformidades ósseas) (MOTTA et al., 2018).

O efetivo controle da sífilis tem como premissa fundamental a triagem sorológica e o tratamento adequado de gestantes e parceiros sexuais, visto que a qualidade da 
assistência pré-natal e ao parto é um importante determinante na redução da transmissão vertical. A penicilina é o fármaco de primeira escolha no tratamento da sífilis e o único indicado para gestantes: apresenta $98 \%$ de eficácia na prevenção da sífilis congênita, agindo em todos os estágios da doença (CAVALCANTE; PEREIRA; CASTRO, 2017).

\section{OBJETIVO}

Apresentar o número de casos notificados de sífilis congênita na região norte do Brasil, entre os anos de 2014 a 2019, e caracterizar o perfil epidemiológico dos casos.

\section{MÉTODO}

Realizou-se um estudo quantitativo descritivo, transversal e retrospectivo através de dados do Sistema de Informações de Agravos de Notificação (SINAN/SUS) do Departamento de Informática do Sistema Único de Saúde - DATASUS. Foram coletados dados nacionais de acordo com as seguintes etapas: Acessou-se o link http://indicadoressifilis.aids.gov.br e em "Abrangência de dados" selecionou-se "Dados regionais e nacionais" e em "Subcategoria" "Brasil". Clicou-se no botão "Baixar Dados" e retirou-se os dados referentes a sífilis congênita Brasil. Posteriormente em "Abrangência de dados" selecionou-se "Dados Estaduais" e em "Subcategoria" foi-se selecionando o nome de cada estado da região norte do Brasil, as tabelas de cada estado foram baixadas e as informações a sífilis congênita retiradas. A compilação dos dados foi feita dentro do aplicativo Excel, componente do pacote Office da Microsoft Corporation.

\section{RESULTADOS}

O percentual de casos de sífilis congênita segundo diagnóstico final por ano de diagnóstico no Brasil entre 2014 e 2019 está representado na tabela 1. Observa-se um aumento na porcentagem de casos de 12,28\% em 2014 para 18,21\% em 2019. 
Tabela 1 Mostra o percentual de casos de sífilis congênita segundo diagnóstico final por ano de diagnóstico, na região Norte do Brasil, entre 2014 e 2019.

\begin{tabular}{rrc}
\hline ANO & No CASOS & $\%$ \\
\hline $\mathbf{2 0 1 4}$ & 16353 & $12.28 \%$ \\
$\mathbf{2 0 1 5}$ & 19712 & $14.80 \%$ \\
$\mathbf{2 0 1 6}$ & 21330 & $16.01 \%$ \\
$\mathbf{2 0 1 7}$ & 25037 & $18.79 \%$ \\
$\mathbf{2 0 1 8}$ & 26531 & $19.92 \%$ \\
$\mathbf{2 0 1 9}$ & 24253 & $18.21 \%$ \\
\hline
\end{tabular}

$\mathrm{Na}$ região Norte do Brasil o percentual de casos de sífilis congênita segundo diagnóstico final por ano de diagnóstico, entre 2014 e 2019, foi de 11.071. No período, houve crescimento do número de casos, de $11,13 \%$ no ano de 2014 para $20,19 \%$ em 2019 (Figura 1).

Figura 1 Mostra o percentual de casos de sífilis congênita segundo diagnóstico final por ano de diagnóstico, na região Norte do Brasil, entre 2014 e 2019.

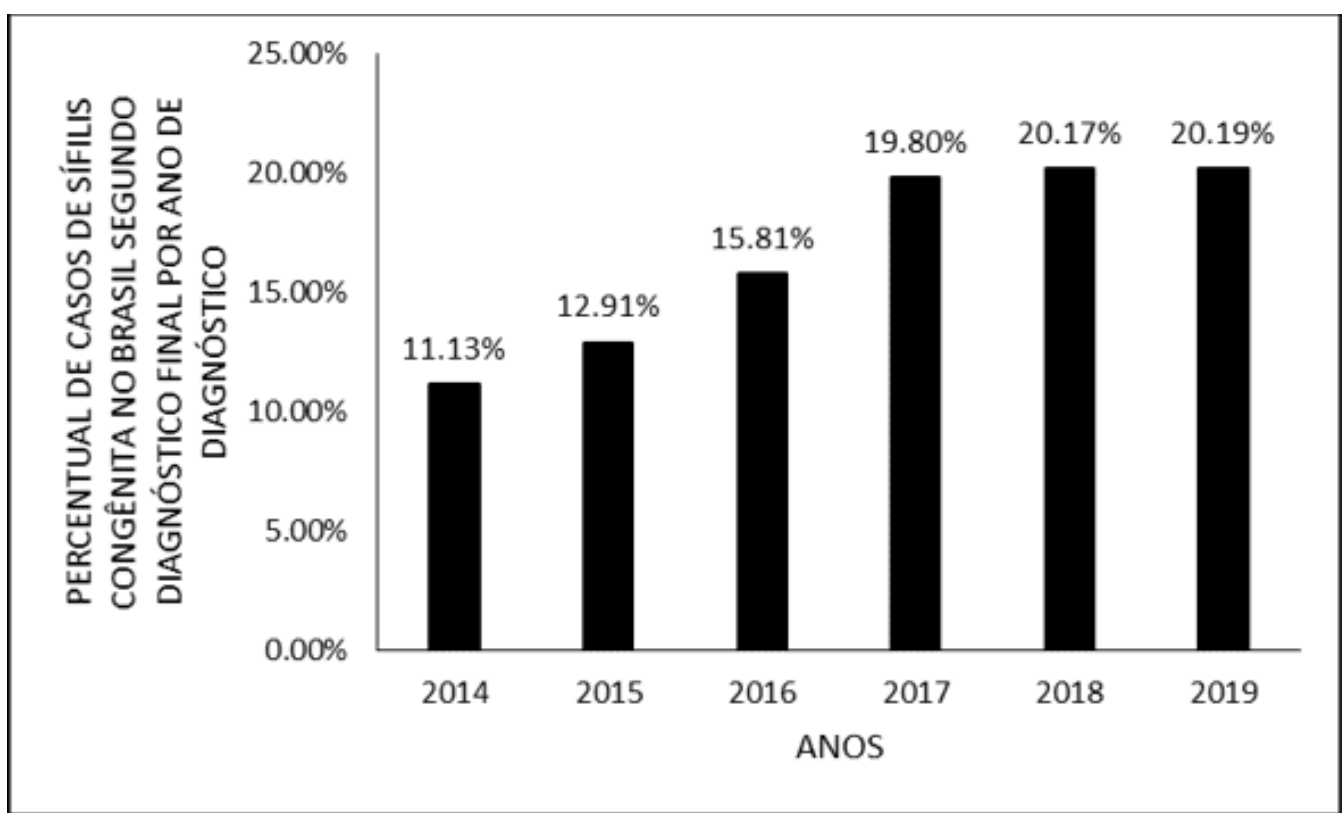


Dentre os Estados da região Norte, o Pará destacou-se com o maior número de casos $(42,08 \%)$ e o com menor montante, o estado de Roraima (1,83\%). (Figura 2$)$.

Figura 2 Mostra o percentual de casos de sífilis congênita segundo diagnóstico final por ano de diagnóstico, por estado da região Norte do Brasil, entre 2014 e 2019.

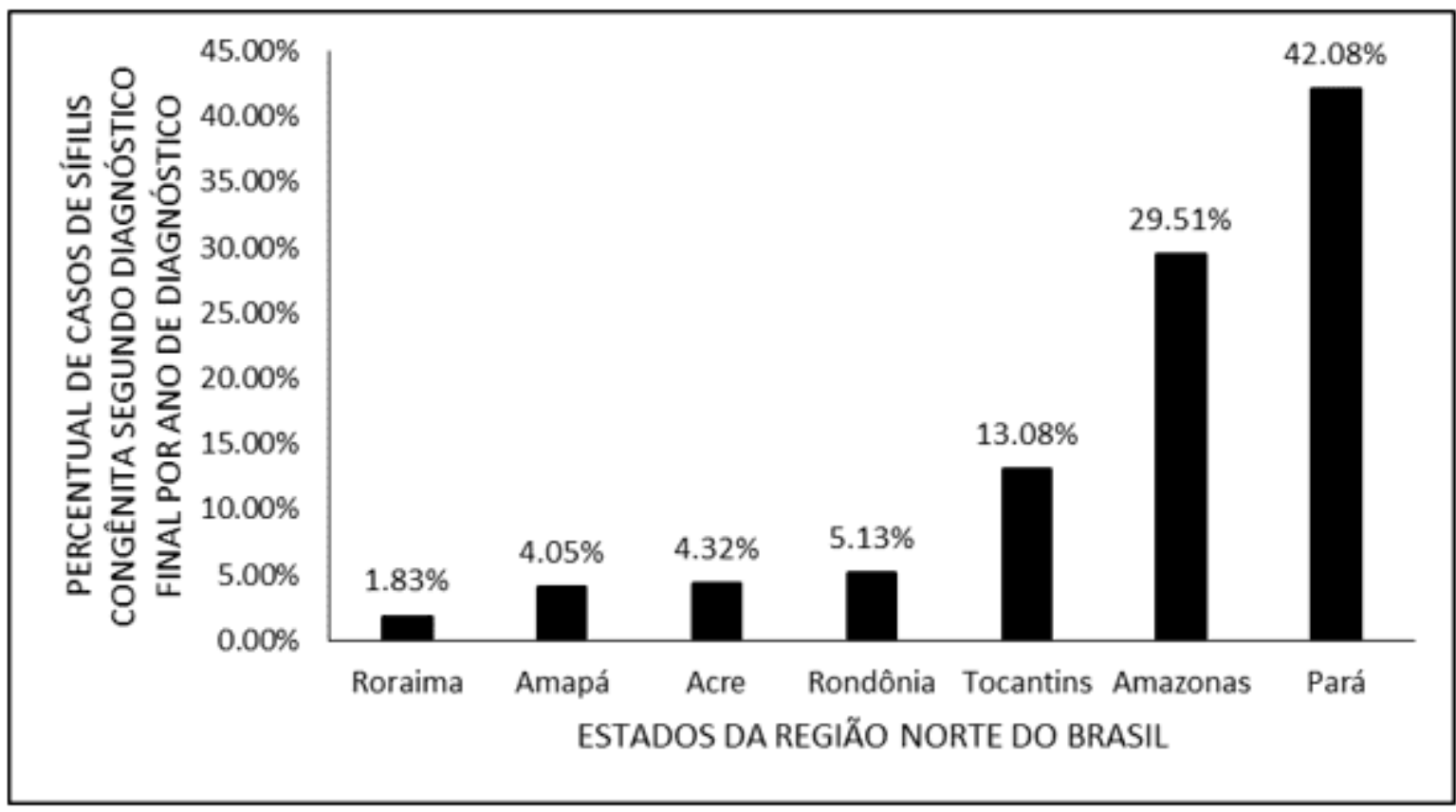

A Figura 3 mostra a distribuição percentual de casos de sífilis congênita na região Norte por segundo raça/cor (etnia) entre os anos de 2014 e 2019. Os dados indicam uma quantidade maior no número de casos na etnia parda com $9.638(87,06 \%)$, seguida pela branca com $608(5,49 \%)$. As etnias que apresentaram menor número foram indígenas com $85(0,77 \%)$ e preta com $32(0,29 \%)$, respectivamente. 
Figura 3 Mostra a distribuição percentual de casos de sífilis congênita na região Norte por segundo raça/cor (etnia) entre os anos de 2014 e 2019.

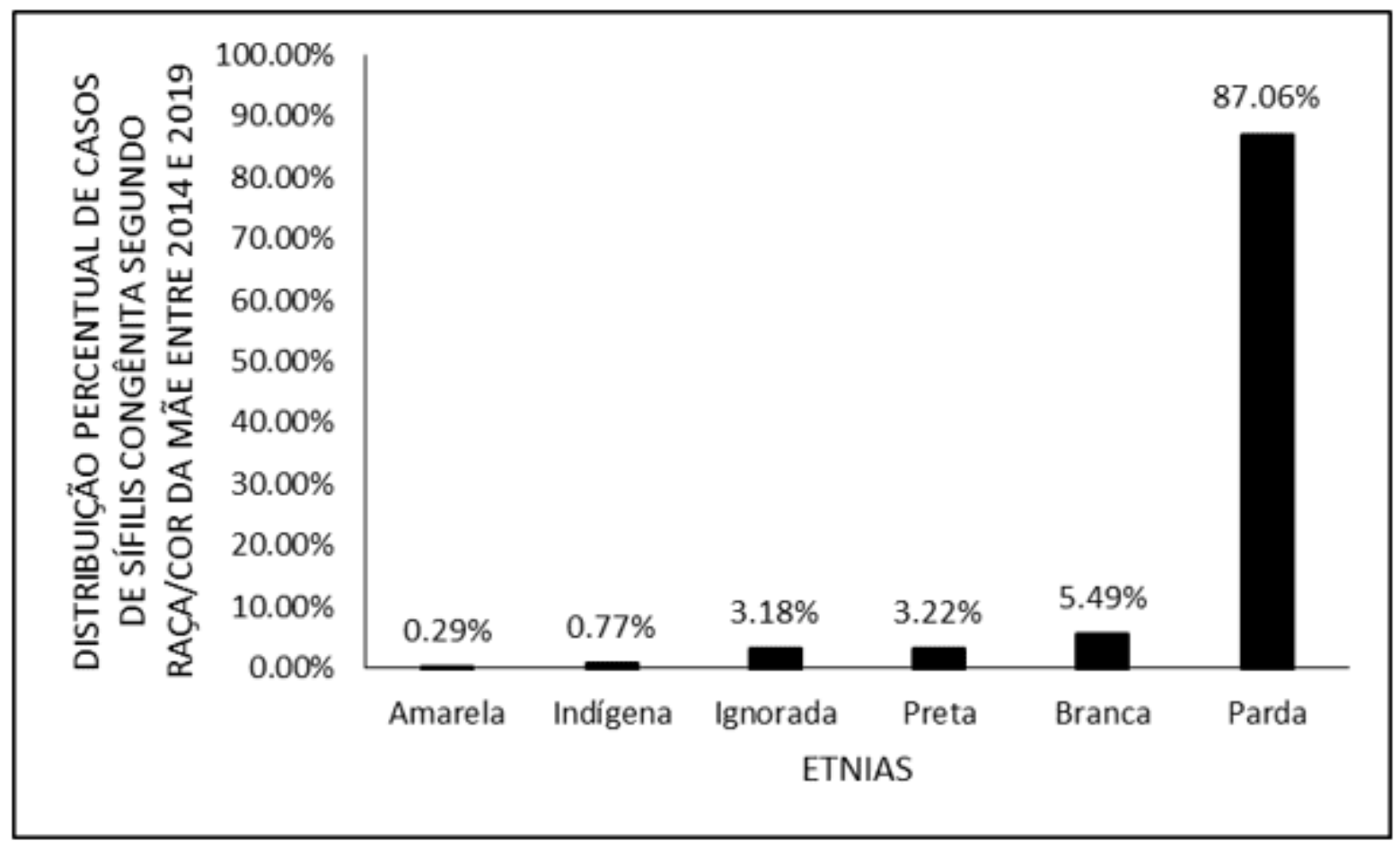

A figura 4 mostra o número de casos confirmados de sífilis congênita na região Norte por realização de exame pré-natal entre os anos de 2014 e 2019. Os dados mostram uma quantidade maior de mulheres que realizaram o pré-natal $8.712(78,69 \%)$ do que as que não realizaram $2.048(18,50 \%)$ e aquelas consideradas ignoradas totalizaram $311(2,81 \%)$. 
Figura 4 mostra a distribuição percentual de casos de sífilis congênita segundo informação sobre realização de pré-natal da mãe por ano de diagnóstico entre 2014 e 2019.

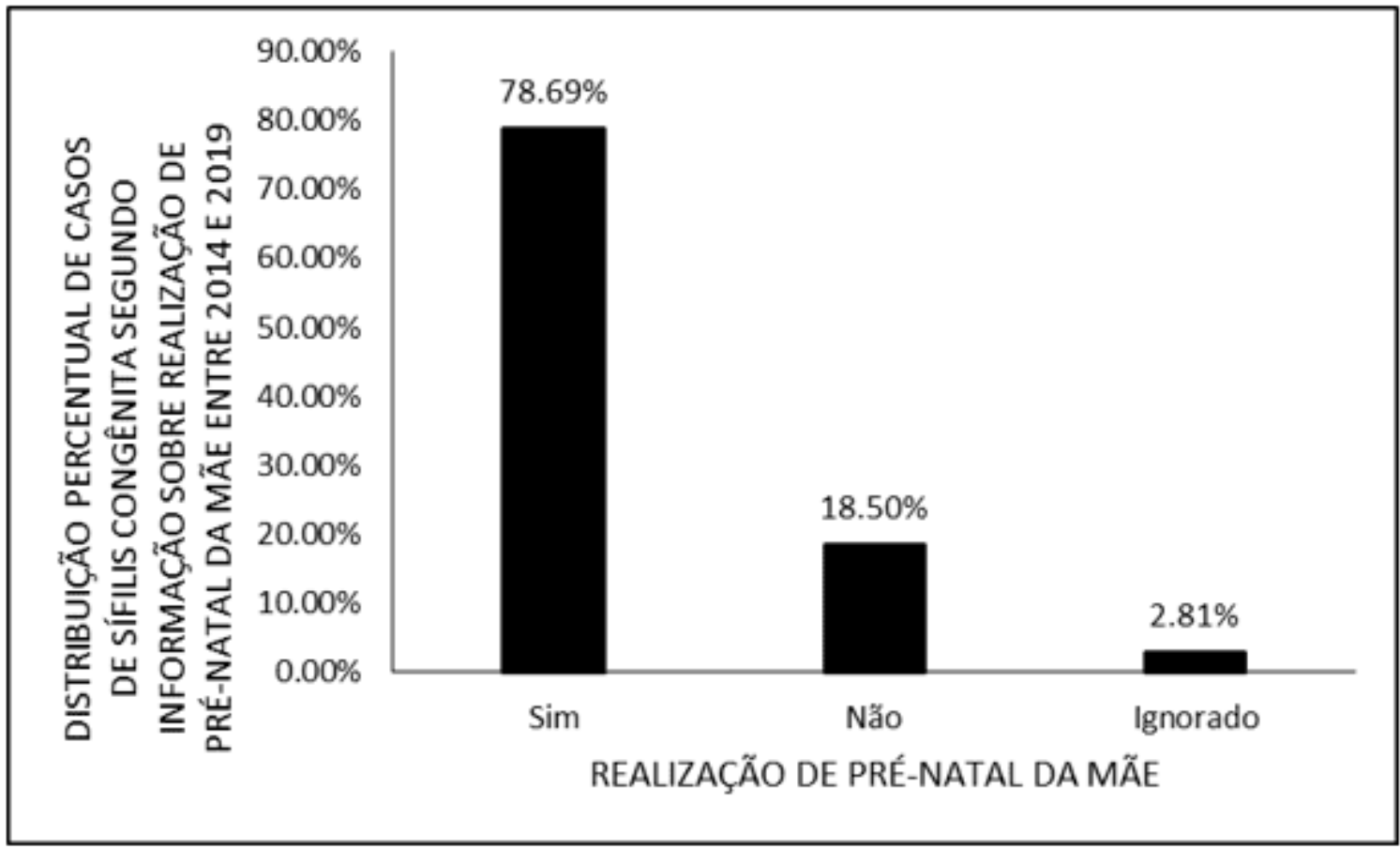

A figura 5 mostra a distribuição percentual de casos de sífilis congênita na região Norte, segundo escolaridade da mãe por ano de diagnóstico entre os anos de 2014 e 2019. Os dados mostram um quantitativo importante de mães analfabetas de 62 mulheres $(0,90 \%)$, sendo a maioria de casos com o grau escolar materno da $5^{\underline{a}}$ a $8^{\mathrm{a}}$ série incompleta do ensino fundamental com 2.362 (33,80\%), com um acervo de mulheres tendo completado o ensino fundamental de $936(13,40 \%)$ e uma parte completou o ensino médio 1.320 (18,90\%), sendo o menor número de mães com a educação superior completa $62(0,90 \%)$ e o quantitativo de ignorados de 2.243 $(32,10 \%)$. 
Figura 5 Mostra a distribuição percentual de casos de sífilis congênita na região Norte, segundo escolaridade da mãe por ano de diagnóstico entre os anos de 2014 e 2019.

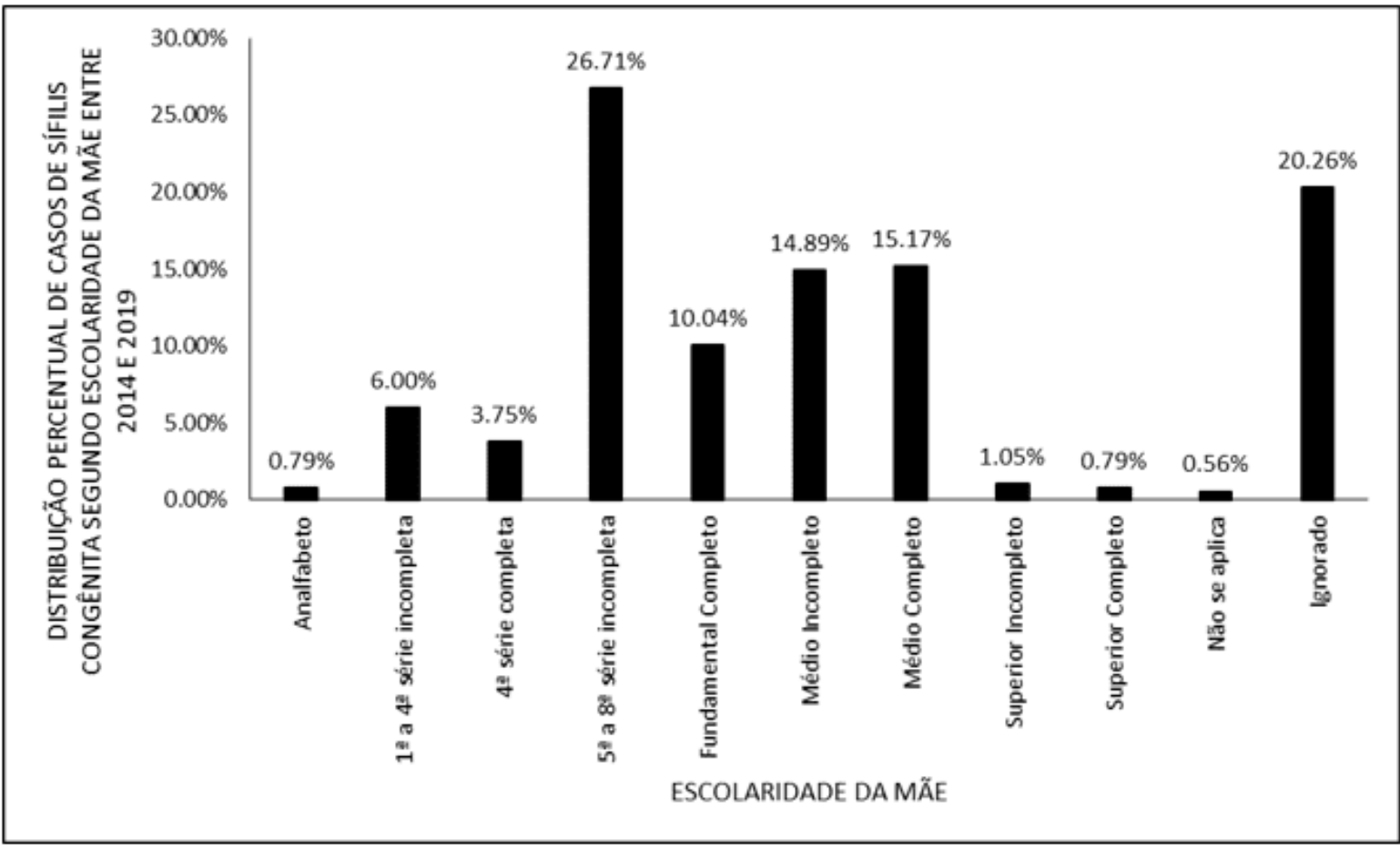

A figura 6 mostra o número de casos confirmados de sífilis congênita na região Norte pelo momento do diagnóstico materno por sífilis, entre os anos de 2014 e 2019. Percebe-se uma maior quantidade de infecções durante o exame pré-natal com total de casos de $4.862(43,92 \%)$ e também ao momento do parto com $4.258(39,46 \%)$, sendo a minoria de casos diagnosticados no pós-parto, com total de $1388(12,54 \%)$ e os ignorados totalizaram 447 (4,04\%). 
Figura 6 Mostra a distribuição percentual de casos confirmados de sífilis congênita segundo o momento de diagnóstico na região Norte, entre os anos de 2014 e 2019.

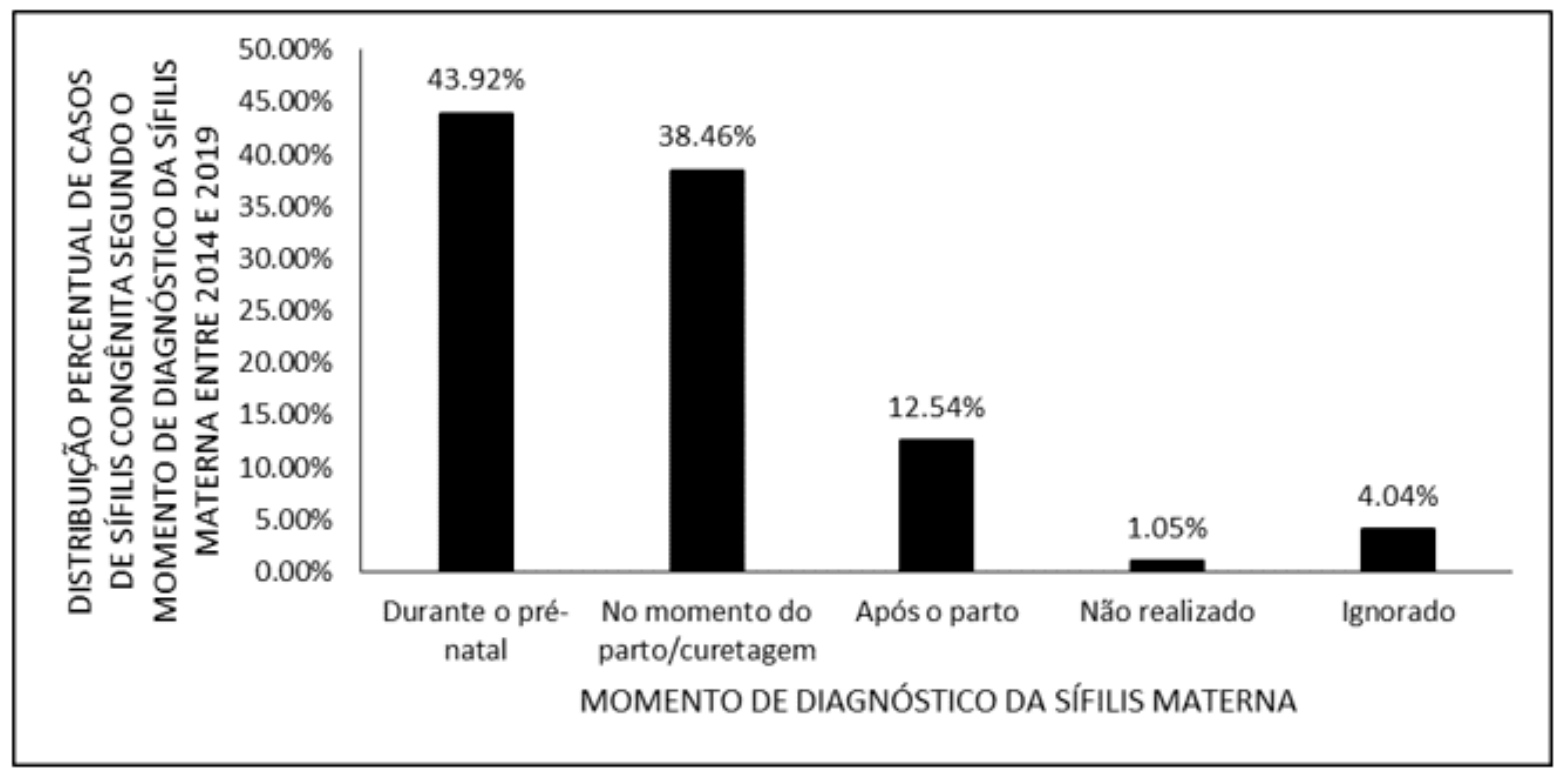

Ao que se refere à variável classificação final da sífilis congênita, os dados encontrados foram de 10.475 (94,62\%) classificada como sífilis congênita recente; 24 classificadas como sífilis congênita tardia (0,22\%); 256 (2,31\%) abortos por sífilis; e $316(2,85 \%)$ natimortos por sífilis (Tabela 2$)$.

Tabela 2 Mostra a distribuição percentual de casos de sífilis congênita segundo diagnóstico final por ano de diagnóstico, na região Norte do Brasil, entre 2014 e 2019.

\begin{tabular}{c|c|c}
\cline { 2 - 3 } & $\begin{array}{c}\text { No CASOS } \\
\text { (2014 a 2019) }\end{array}$ & $\%$ \\
\hline $\begin{array}{c}\text { Sífilis congênita } \\
\text { recente }\end{array}$ & 10475 & $94.62 \%$ \\
Sífilis congênita tardia & 24 & $0.22 \%$ \\
Aborto por sífilis & 256 & $2.31 \%$ \\
Natimorto por sífilis & 316 & $2.85 \%$ \\
\hline
\end{tabular}




\section{DISCUSSÃO}

Houve um aumento do número de casos dos anos de 2014 até 2019 (figura 1), com destaque para o Estado do Amazonas que aumentou em 416\% (figura 2) os registros de notificação da doença no período observado, mesmo sendo, o Estado da Região Norte com o maior PIB per capita (IBGE, 2016). Essa análise da relação da incidência da doença com a capacidade econômica do local é feita pelo fato da sífilis congênita ser uma doença de diagnostico relativamente fácil e de ser totalmente evitável quando o tratamento da gestante e do parceiro é realizado de maneira adequada (ARAUJO et al., 2006), o que faz a sua prevalência ser geralmente relacionada a um poder econômico deficiente para se disponibilizar uma Saúde Básica de qualidade, mais especificamente, para oferecer um Pré-Natal efetivo para a população (ARAUJO et al., 2006).

Porém, o subdesenvolvimento não explicaria sozinho a causa do Amazonas ser o principal Estado em crescimento da doença no período observado, pois comparado aos outros Estados da Região Norte, é o de melhor PIB, assim a melhora da notificação da doença no Estado, poderia explicar o fato da Região Norte apresentar prevalência alta dessa comorbidade. Em 2016, a Secretaria de Saúde do Amazonas iniciou uma campanha de descentralização da realização de testes rápidos para doenças sexualmente transmissíveis, antes superconcentrado na FMT (Fundação de Medicina Tropical Heitor Vieira Dourado), redistribuindo-os pelos quatro SAEs (Serviços de Atenção Especializada) da cidade (BRASIL, 2016).

Ainda sobre as perspectivas que buscam explicar a alta incidência da sífilis congênita na região Norte, o subdesenvolvimento também está relacionado a uma população com menor nível de conscientização, ou seja, não seria suficiente apenas a disponibilização do pré-natal, mas o entendimento da sociedade acerca da importância da sua realização, o que ajuda a entender porque é um problema de saúde pública ainda tão presente na Região Norte do Brasil, a segunda região brasileira com o menor PIB per capita, perdendo apenas para o Nordeste (IBGE, 2016). 
Acerca da variável etnia nos casos registrados de sífilis congênita (figura 3), mais de $90 \%$ dos casos era de etnia (raça) parda que também é a etnia mais prevalente na Região Norte, representando $66,88 \%$ da população dessa região do Brasil (IBGE, 2010).

É importante também traçar um paralelo com dois dados levantados na pesquisa, a porcentagem de mães infectadas que realizaram pré-natal é de mais de $70 \%$, no período observado (2014-2019) (figura 4), ou seja, a maioria das mulheres na região fazem esse acompanhamento o que deveria implicar numa incidência menor da doença. Em contrapartida, o número de parceiros não tratados é alto. A sífilis é uma doença de transmissão sexual, onde é, mandatório o tratamento do parceiro. Isso reflete uma difícil realidade do Brasil na realização de um pré-natal efetivo, a falta do acompanhamento satisfatório do parceiro nas consultas. Um dos principais problemas que ainda existe no controle das infecções sexualmente transmissíveis (IST's) durante a gravidez é a abordagem do parceiro (DUARTE, 2007). Acrescentando a isso, os problemas surgem com a notificação e continuam na tentativa de convencê-los da necessidade diagnóstica e de adesão ao tratamento (DUARTE, 2007).

Outro fator é o nível de analfabetismo, apenas 0,9\% eram analfabetas e, a maior porcentagem é de pessoas que concluíram no mínimo o ensino fundamental (figura 5). Porém, cerca de $8 \%$ da população do norte do Brasil é analfabeta, segunda pior região, perdendo apenas para a Região Nordeste (IBGE, 2019). Essa comparação de dados, leva ao questionamento acerca da subnotificação, se a maior porcentagem de mulheres com sífilis na região de fato tem escolaridade maior ou se a porcentagem que procura atendimento e tem os casos notificados é a maioria instruída e a que tem menor instrução segue sem procurar atendimento e não tem a doença notificada.

Um pré-natal inadequado impede a realização da rotina pro diagnóstico e o tratamento efetivo e precoce (ARAUJO et al, 2006). Os dados sobre o momento do diagnóstico reforçam a importância do pré-natal efetivo para combater a incidência da sífilis congênita, pois a maioria teve o diagnóstico no parto ou no momento da curetagem (figura 6). 


\section{CONCLUSÃO}

Pela alta incidência dos casos de Sífilis Congênita há o destaque da região Norte em detrimento das outras regiões brasileiras. O subdesenvolvimento da região é fator fundamental para a explicação desse fato, quando há um déficit nos setores públicos, principalmente na educação e na saúde, as evidências são repercutidas na população. Em relação à Sífilis Congênita, a maioria das gestantes tinha um grau de escolaridade baixo, diante desse dado, infere-se uma falta de informação sobre a importância do pré-natal, tanto pela falta de interesse da população como a pouca divulgação do sistema público de saúde. Como exemplo, o estado do Amazonas que foi o estado com a maior taxa percentual de casos notificados no período analisado por conta de uma maior atuação no rastreio de infecções sexualmente transmissíveis, porém, a maior disponibilidade dos métodos de rastreio, exclusivamente, não é sinônimo de uma melhora na incidência dos casos de Sífilis Congênita.

Ainda, a quantidade de parceiros não tratados tem grande relevância, já que a via de transmissão mais comum da Sífilis é a via sexual. Existe a falta de informação e resistência pela maioria deles. Dessa forma, existe a necessidade da gestante em conjunto com seu parceiro serem rastreados durante o pré-natal para evitar uma possível reinfecção de uma paciente tratada ou diagnóstico precoce.

Diante disso, observa-se que para a diminuição da incidência de casos de Sífilis Congênita é necessária uma gama de fatores, desde a maior disponibilidade de informação sobre a importância da realização do pré-natal e sua continuidade por parte das gestantes e seus parceiros. Com isso, abranger o diagnóstico e tratamento precoces, afim de evitar complicações para o feto ou neonato.

\section{REFERÊNCIAS}

ARAUJO, Eliete da Cunha; Costa, Kelly de Souza Gama; SOUZA E SILVA, Rafaela; AZEVEDO, Valéria Nascimento; Lima, Fábio André Souto. Importância do pré-natal na prevenção da Sífilis Congênita. Revista Paraense de Medicina, Belém, v. 20, n. 1, p. 47-51, mar. 2006.2 Disponível 
em:

http://scielo.iec.gov.br/scielo.php?script=sci arttext\&pid=S010159072006000100008\&lng=pt\&nrm=iso Acesso em: 24 maio 2019.

BARROS, Yara Lorrane Souza de. Et al. Numbers of confirmed syphilis cases in pregnant women in Brazil between 2009 and 2013. Revista Científica Multidisciplinar Núcleo do Conhecimento. Year 05, Ed. 11, Vol. 25, pp. 53-61. November 2020. ISSN:2448-0959, Access link in: https://www.nucleodoconhecimento.com.br/health/syphilis-cases, DOI: 10.32749/nucleodoconhecimento.com.br/health/syphilis-cases

BRASIL, Ministério da Saúde. Departamento de Doenças de Condições Crônicas e Infecções Sexualmente Transmissíveis: Manaus recebe apoio da ONG AHF para descentralizar 0 atendimento de HIV/aids. 2016. Disponível em: http://www.aids.gov.br/pt br/noticias/manaus-recebe-apoio-da-ong-ahf-paradescentralizar-o-atendimento-de-hivaids _ Acesso em: 23 maio 2019.

BRASIL, Ministério da Saúde. Departamento de Vigilância, Prevenção e Controle das Infecções Sexualmente Transmissíveis, do HIV/Aids e das Hepatites Virais: Protocolo Clínico e Diretrizes Terapêuticas para Atenção Integral às Pessoas com Infecções Sexualmente Transmissíveis (IST). 2018. Disponível em: http://conitec.gov.br/images/Artigos Publicacoes/Diretrizes/PCDT Atencao Integral IST 22-10-18.pdf Acesso em: 23 maio 2019.

CARDOSO, Ana Rita Paulo; ARAÚJO, Maria Alix Leite; CAVALCANTE, Maria do Socorro; FROTA, Mirna Albuquerque; DE MELO, Simone Paes. Análise dos casos de sífilis gestacional e congênita nos anos de 2008 a 2010 em Fortaleza, Ceará, Brasil. Ciência \& Saúde Coletiva. v. 23, n. 2, p. 563-574, 2018. Disponível em: https://www.scielosp.org/article/csc/2018.v23n2/563-574/ Acesso em: 24 maio 2019.

CAVALCANTE, Ana Egliny S; SILVA, Maria Adelane M; RODRIGUES, Antonia Regynara M; NETTO, José Jeová Mourão; MOREIRA, Andréa CA; GOYANNA, Natália F. Diagnóstico e tratamento da sífilis: uma investigação com mulheres assistidas na atenção básica em Sobral, Ceará. DST Jornal Brasileiro de Doenças 
Sexuais. v. 24, n. 4, p. 245-245, 2012. Disponível em: http://www.dst.uff.br/revista244-2012/4-Diagnostico\%20e\%20Tratamento\%20da \%20Sifilis.pdf Acesso em: 23 maio 2019.

CAVAlCANTE, Patricia Alves de Mendonça; PEREIRA, Ruth Bernardes de Lima; CASTRO, Jose Gerley Diaz. Sífilis gestacional e congênita em Palmas, Tocantins, 2007-2014. Epidemiologia e Serviços de Saúde. v. 26, n. 2, p. 255-264, abr./jun, 2017. Disponível em: https://www.scielosp.org/article/ress/2017.v26n2/255264/pt/ Acesso em: 23 maio 2019.

CRT. Centro de Referência e Treinamento DST/AIDS-SP. Coordenadoria de Controle de Doença, Secretaria de Estado da Saúde de São Paulo (SES-SP). Guia de bolso para o manejo de sífilis em gestante e sífilis congênita. 2 ed., 112 p. São Paulo: Secretaria de Estado da Saúde. 2016. Disponível em: http://www.saude.campinas.sp.gov.br/saude/doencas/sifilis/guiadebolsodasifilis_2edi cao2016.pdf Acesso em: 23 maio 2019.

DUARTE, Geraldo. Extensão da Assistência Pré-natal ao Parceiro como Estratégia de Aumento da adesão ao Pré-natal e Redução da Transmissão Vertical de Infecções. Revista Brasileira de Ginecologia e Obstetrícia. v. 29, n. 4, p. 171-174, 2007.

GUINSBURG, Ruth; SANTOS, Amélia Miyashiro Nunes dos Santos. Critérios diagnósticos e tratamento da sífilis congênita. Documento científico Departamento de Neonatologia. São Paulo: Sociedade Brasileira de Pediatria. 2010. Disponível em: https://www.sbp.com.br/fileadmin/user upload/2015/02/tratamento sifilis.pdf Acesso em: 24 maio 2019.

Instituto Brasileiro de Geografia e Estatística (IBGE). Contas regionais do Brasil: 2010-2014, Coordenação de Contas Nacionais. - Rio de Janeiro: IBGE, 2016. 97 p. (Contas nacionais, ISSN 1415-9813; n. 53). Disponível em: https://biblioteca.ibge.gov.br/visualizacao/livros/liv98881.pdf Acesso em: 24 maio 2019. 
Instituto Brasileiro de Geografia e Estatística (IBGE), Diretoria de Pesquisas, Coordenação de Trabalho e Rendimento, Pesquisa Nacional por Amostra de Domicílios Contínua 2016-2018. 2019. Disponível em: https://biblioteca.ibge.gov.br/visualizacao/livros/liv101657_informativo.pdf Acesso em: 24 maio 2019.

IBGE. Instituto Brasileiro de Geografia e Estatística. Sistema IBGE de Recuperação Automática - $\quad$ SIDRA. 2010.2 Disponível em: https://sidra.ibge.gov.br/Tabela/3175\#resultado Acesso em: 24 maio 2019.

MOTTA, Isabella almeida; DELFINO, Isabella Rey de Souza; DOS SANTOS, Leticia Vettorazzi; MORITA, Maura Omori; GOMES, Rayanne Gonçalves Dantas; MARTINS, Talita Pouzas Soares; CARELLOS, Ericka Vianna Machado; ROMANELLI, Roberta Maia de Castro. Sífilis congênita: por que sua prevalência continua tão alta?. Revista Médica de Minas Gerais, Belo Horizonte, v. 28. Suppl. 6, 2018. Disponível em: http://rmmg.org/artigo/detalhes/2418 Acesso em: 23 maio 2019.

ROCHA, Karina Dias. Et al. Number of congenital syphilis cases in Brazil between 2009 and Revista Científica Multidisciplinar Núcleo do Conhecimento. Year 05, Ed. 05, Vol. 01, pp. 131-143. May 2020. ISSN:2448-0959 DOI: 10.32749/nucleodoconhecimento.com.br/health/congenital-syphilis-cases

Enviado: Dezembro, 2020.

Aprovado: Dezembro, 2020. 